\title{
Del repudio a los malos profesores a la emancipación social. Los reclamos de los estudiantes porteños (1872-1930)
}

\author{
Natalia Bustelo \\ Cedinci - Unsam \\ nataliabustelo@yahoo.com.ar
}

\section{Reclamos estudiantiles}

Como una forma de conmemorar el centenario del estallido de la Reforma Universitaria, las páginas que siguen vuelven una vez más sobre los primeros conflictos estudiantiles registrados en la Universidad de Buenos Aires, pero su propósito es revisar una cuestión que permanece poco atendida: la vinculación que tramaron los distintos conflictos con el orden político. El artículo se divide en cuatro apartados. Cada uno de ellos analiza la dimensión gremial y la política de una iniciativa estudiantil identificada con la "reforma universitaria". La sucesión de iniciativas permite identificar la configuración de una agenda de reclamos gremiales, pero también sugiere que si 1918 marca una ruptura en la sociabilidad estudiantil es porque una minoria de estudiantes consiguió fundar un movimiento que alejaba a esa agenda gremial de una república oligárquica para sumarla, desde distintas posiciones, a la lucha emancipatoria librada por las izquierdas.

\section{Reforma universitaria para perfeccionar a la elite oligárquica}

Hacia 1860 el territorio argentino superaba las prolongadas guerras civiles y avanzaba en la consolidación de una República oligárquica. La elite político-económica que gobernaría la Argentina entre 1860 y 1916 mostraba su capacidad para superar la profunda crisis económica y política de 1890. Desde entonces deberia hacer frente a una creciente conflictividad social y política, pero ello no le impedia proseguir la construcción de un orden social laico que en el plano político negaba la ampliación democrática mientras que lograba una importante mo-

(Archivos, año VI, $\mathrm{n}^{\circ}$ 12, marzo de 2018, pp. 33-52) 
dernización económica. Esta se estructuraba en nuevas formas de desigualdad y opresión capitalistas. Los pilares de la economía comenzaban a ser la masiva llegada de trabajadores europeos, la agricultura latifundista-que desplazaba a las comunidades originarias y al campesinado al tiempo que profundizaba la desigualdad entre las regiones-, la inversión neocolonial y la inserción del mercado local en el sistema capitalista mundial. ${ }^{1}$

Ese desarrollo requería, entre otras cosas, la regulación de tres actividades: la medicina, la ingeniería y la abogacía. La Universidad de Buenos Aires y la Universidad de Córdoba, sobre todo sus facultades de derecho, funcionaban desde hacía varias décadas como los ámbitos de sociabilidad de la elite política, que luego de formarse en la nueva república partía al "viaje de iniciación" europeo (Viñas, 1964: 46-51). Las universidades además eran las únicas habilitadas para expedir matrículas profesionales. Como subraya Pablo Buchbinder (2005: 6061), entonces se iniciaba la prolongada discusión -que continúa en el presente- sobre el perfil de las universidades: una parte importante de la elite gobernante bregaba por su consolidación como formadoras de los profesionales liberales mientras que una fracción minoritaria apostaba a universidades orientadas a la investigación científica.

Antes de que el parlamento sancionara las distintas leyes relativas al sistema educativo argentino, los estudiantes impugnaban a las autoridades de la Universidad de Buenos Aires y se hacian visibles en las calles. El 13 de diciembre de 1871 el joven Roberto Sánchez se suicidaba luego de ser reprobado injustamente en un examen de la Facultad porteña de Derecho. Al entierro asistieron más de dos mil personas. Al año siguiente doscientos jóvenes se reunieron en asamblea para fundar la Asociación 13 de Diciembre, crear una Junta Revolucionaria pro Reforma Universitaria y comenzar a editar los periódicos mensuales 13 de Diciembre y El Estudiante. Las doce páginas de cada entrega les permitieron a los estudiantes difundir noticias universitarias así como denuncias y pronunciamientos relativos a la Universidad de Buenos Aires, pero los costos del financiamiento y la distribución pronto truncaron ambos proyectos.

El primer manifiesto de la Asociación colocaba en el centro de su denuncia la parcialidad con que los profesores examinaban a los estudiantes. Se lee allí:

1. Para caracterizar los regímenes políticos argentinos, retomamos a lo largo del artículo las expresiones "República oligárquica" y "República democrática" utilizadas, entre otros, por Botana y Gallo (1997). Pero a ellas agregamos el énfasis sobre las formas de desigualdad y opresión capitalista estructuradas por ambas repúblicas así como el carácter liberal y restringuido de la dimensión democrática (Adamovsky, 2009). 
La mayor parte de los catedráticos dan lecciones particulares en sus casas habitaciones, lecciones a precio de oro, a las que asisten los discipulos de la Universidad que quieren propiciarse la buena voluntad del catedrático para el examen próximo. (Citado en Ortiz y Scotti, s/d: 14)

Durante 1872 la Asociación elevó distintos petitorios a las autoridades universitarias para reformar los estatutos y el régimen de exámenes. Sus reclamos consiguieron, además de la renuncia de varios profesores cuestionados por su escasa formación, que el entonces gobernador de Buenos Aires, Emilio Castro, le explicara al rector universitario, Juan María Gutiérrez, algo que debería ser obvio en todo sistema de enseñanza. En carta pública Castro sostenía que los catedráticos estaban inhabilitados para "dar lecciones o repasos a los alumnos matriculados en la Universidad, sea en otros colegios o en sus propias casas, recibiendo por ello un estipendio o compensación”. Las páginas de El Estudiante aclaraban que los asociados no se ocupaban de politica, sino de las "aspiraciones tendientes al perfeccionamiento de la enseñanza, a la introducción de los buenos sistemas, a la vulgarización de las verdades científicas y al progreso de la literatura". Dios, patria, familia y bello sexo eran señalados como sus valores. ${ }^{2}$

Si bien las autoridades universitarias anunciaron que los estudiantes que cometieran actos de indisciplina serian sancionados con la inhabilitación para inscribirse en las materias -o incluso con la expulsión de la universidad-, las protestas no cuestionaban el lugar de las universidades en la República oligárquica. Se trataba de una juventud -compuesta, en su mayoría, por hijos de familias patricias- que con esos reclamos asumía tempranamente la condición de elite conductora de la República y sus instituciones. La prueba más clara de ello es que poco después Estanislao Zeballos y otros líderes de la Asociación se erigían en destacados miembros de esa generación del 80 que gobernó el país hasta 1916.

Para que los pocos estudiantes porteños de ingeniería expresaran conjuntamente sus reclamos deberian pasar algunos años. A la incipiente organización de derecho siguió la de los estudiantes de la Facultad de Medicina, quienes en 1875 fundaron el Círculo Médico Argentino, una iniciativa que, al igual que la Asociación 13 de Diciembre, fue liderada por un futuro miembro de la generación del 80, el joven José María Ramos Mejía. En sus inicios el Círculo congregó a la mitad de los estudiantes, emprendió campañas en la prensa contra algunos cursos y otras cuestiones gremiales y fundó una biblioteca (Halperín Donghi,

2. "Nuestros propósitos", El Estudiante, n 1, 4 de marzo de 1872, p. 1. 
1962: 82-84). En 1877 sumó la edición de los Anales del Círculo Médico Argentino y poco después, cuando sus fundadores habian dejado la condición de estudiantes, el Círculo comenzó a expresar los reclamos corporativos de los médicos. Entrado el siglo XX, las asociaciones estudiantiles encargadas de los reclamos gremiales se convertirian en Centros de Estudiantes con personería jurídica.

Además de tramarse esa sociabilidad estudiantil, en las últimas décadas del siglo XIX ingresaban las primeras mujeres a las carreras de la Facultad de Medicina. Ese ingreso debia sortear las resistencias del Consejo Académico y de muchos estudiantes. Varias mujeres fundaron asociaciones feministas que, ligadas al socialismo, el anarquismo o el librepensamiento, reclamaron la admisión universitaria y, en general, la igualdad de derechos. Entre esas asociaciones se destacan el Consejo Nacional de Mujeres, creado en 1901, y Universitarias Argentinas, asociación organizadora en 1910 de un masivo congreso femenino internacional (Barrancos, 2007: 132-134). A pesar de esa trama, serían muy pocas las mujeres que participarian de los Centros de Estudiantes y de los reclamos por las diversas reformas universitarias. Y ello al punto que, en su despliegue nacional, el movimiento de 1918 no registró entre sus reclamos el derecho de las mujeres a recibir formación universitaria ni contó con una líder mujer.

En definitiva, con la Joven Argentina o generación del 37, que tuvo en Echeverria, Alberdi y Sarmiento a sus más destacados representantes, se habia iniciado en Argentina un extendido asociacionismo juvenil de carácter político. A lo largo del siglo XIX este se manifestó en las ramas juveniles de los clubes políticos y en el siguiente en las juventudes de los partidos políticos. Con la Asociación 13 de Diciembre se inauguraba, en cambio, un juvenilismo que se pronunciaba sobre las cuestiones gremiales de los estudiantes y que, a pesar de su declarada apoliticidad, enlazaba la reforma universitaria a una mejor formación de una elite oligárquica, sobre la que no se dudaba que estaba destinada a dirigir el país. Y veremos en el tercer apartado que ese juvenilismo gremial y oligárquico alcanzó una escala latinoamericana a través de la Liga de Estudiantes Americanos.

\section{Reforma universitaria para erradicar la falta de autoridad moral}

Los reclamos gremiales de los estudiantes de la Universidad de Buenos Aires volverian a tener un fuerte impulso a fines de 1903, año en que las huelgas, movilizaciones y tumultos estudiantiles obligaban por primera vez a suspender las clases. Entonces la universidad porteña había sumado la Facultad de Filosofia y Letras y nuevas carreras. Su 
matrícula alcanzaba los dos mil estudiantes y con ello cuadriplicaba la matrícula de la universidad cordobesa. ${ }^{3}$

El crecimiento del estudiantado tenía una relación directa con la recuperación económica lograda por la República oligárquica. Una parte de los nuevos sectores medios podía financiar los estudios de algunos de sus hijos. Las aulas universitarias dejaban de ser un espacio exclusivo de la high society para comenzar a estar recorridas por jóvenes varones que buscaban un título de médico -o, en menor medida, de abogado- que les asegurara un ascenso social y económico. Varios de esos jóvenes serían partícipes de la "malquerida bohemia" que, liderada por los poetas Rubén Dario y Leopoldo Lugones, construyó el movimiento literario modernista junto a espacios de sociabilidad distantes de la elite oligárquica. Además serian entusiastas difusores del anarquismo o del socialismo y tendrian un rol clave en la campaña porteña de desprestigio de los profesores integrantes del Consejo Académico de Derecho y del de Medicina.

Las documentadas investigaciones de Juan Suriano (2004) y de Horacio Tarcus (2007b) no dejan dudas de que, a comienzos del siglo XX, las ideas y prácticas anarquistas y socialistas habian alcanzado una amplia circulación en el Río de la Plata. Desde 1896 Buenos Aires contaba con el primer Partido Socialista de América Latina y una imprenta que editaba el periódico La Vanguardia y diversas colecciones de folletos socialistas. Además el movimiento obrero se había organizado en una federación que adoptaba el comunismo anárquico: la Federación Obrera Regional Argentina (FORA), y el anarquismo también se difundía desde el diario La Protesta y una nutrida folletería.

Para mejorar las duras condiciones de trabajo de los obreros urbanos, en noviembre de 1902 la FORA y los sindicatos de tendencia socialista protagonizaban la primera huelga general argentina. Pero el socialismo y el anarquismo interesaban no solo a los obreros, sino también a algunos estudiantes y unos pocos profesores. Los estudiantes identificados con los valores patrióticos de la República oligárquica continuarian siendo mayoría en las asambleas estudiantiles, pero en ellas surgian los primeros líderes distantes de esos valores. ${ }^{4}$ En efecto, el levantamiento que lideró Hipólito Yrigoyen en 1905 contó con el apoyo de varios estudiantes (Rock, 2006: 291-295). Pero ya en 1895 un joven

3. Seguimos en este apartado las reconstrucciones de Halperín Donghi (1962) y Buchbinder (2005).

4. Sobre las manifestaciones callejeras que, en los primeros años del siglo XX, protagonizaron los estudiantes promotores de un patriotismo oligárquico y belicista, véase Rojkind (2012) y Rock (2006: 265-273). Para un análisis de las diferentes modulaciones del nacionalismo de la primera mitad del siglo XX, véase Devoto (2002). 
José Ingenieros fundaba en la Facultad porteña de Medicina un Centro Socialista Universitario (Tarcus, 2007b: 234-238). A ello agreguemos que en sus memorias Manuel Gálvez (2003: 79-157) ofrece una detallada descripción de la bohemia anarquista de la que participó en su época de estudiante y que lo convenció de codirigir entre 1903 y 1905 la revista cultural filoanarquista Ideas. Nuevas precisiones son ofrecidas por Alfredo Bianchi, quien, además de preparar unas breves memorias sobre la bohemia porteña (1935), confiesa en un artículo en el que polemiza con un compañero de esas aventuras juveniles que "en aquellos años de rebelión de 1904 y 1905 [...] íbamos a la Facultad de Letras con el folleto de Malatesta sobre La anarquía en el bolsillo y La Protesta en la mano". ${ }^{5} \mathrm{Y}$ esas diversas referencias sugieren que el anarquismo se entrelazó con la huelga que iniciaron los estudiantes un año después de la primera huelga obrera general.

En septiembre de 1902 el Consejo Académico de la Facultad de Derecho firmó una resolución a través de la cual los exámenes de marzo dejaban de ser una instancia para aprobar las materias cursadas y se incrementaba el arancel que debian pagar los estudiantes. Estos iniciaban la huelga en diciembre de 1903 y lograban que el Consejo anulara su resolución. Pero al año siguiente el Consejo se negaba a correr la fecha de los exámenes en función de los dias perdidos por las protestas y ello motivó reclamos más abarcativas y contundentes. Los estudiantes comenzaron a denunciar la poca actualización de los profesores y con boycots y otras acciones directas reclamaron la renuncia de varios de esos docentes y la renovación de los contenidos. Recuerda el abogado y diplomático Adolfo Bioy (padre del escritor Adolfo Bioy Casares) que cuando se acercó en febrero de 1904 a la Facultad de Derecho encontró un cartel con la frase "cerrado por falta de autoridad moral" y que pronto se sumó a la huelga y los cabildeos para planear una "acción que debía de ser violenta". Refiere Bioy: "Decidimos penetrar subrepticiamente en casas vecinas de la Facultad y, bien armados, acantonarnos en las azoteas contiguas y, en el momento oportuno, hacer fuego contra las autoridades y contra la policía. Sabiamos que habiase dispuesto asegurar la toma de exámenes con una custodia policial en la casa y eso nos había producido indignación. ¡La casa de estudios ocupada por la policia!" (Bioy, 1963: 88).

Entre 1905 y 1906 los reclamos se extendieron entre los estudiantes y profesores de la Facultad de Medicina. Pero si la impugnación anarquista a la autoridad estuvo presente en las protestas estudiantiles de 1903, las de 1905 tendrian entre sus líderes a los referentes del socialismo argentino. El conflicto se renovaba porque el Consejo Académico de

5. Alfredo Bianchi, "Renegados”, Claridad, n² 2, 5 de febrero de 1920. 
Medicina, por un lado, excluyó a un destacado profesor, Julio Méndez, de la terna para cubrir la cátedra de Clínica Médica y, por el otro, fijó el número máximo de estudiantes que cada mesa de exámenes podía aprobar. La huelga estudiantil finalizó en marzo de 1906 cuando el Consejo eliminó la ordenanza sobre los exámenes. En los meses siguientes se iniciaba un encendido debate universitario. A partir de este, los líderes estudiantiles entregaron a las autoridades universitarias un proyecto de reforma mientras que los profesores y líderes socialistas Juan B. Justo y Nicolás Repetto junto a los profesores Federico Texo y Samuel de Madrid presentaron en el Congreso otro petitorio de reformas. Entre los reclamos se encontraba la docencia libre, el examen de estado, la separación de la gestión administrativa y científica, la renovación periódica de los cuerpos dirigentes, la elección de la mayoría de las autoridades más importantes de la Universidad y la concesión de derechos electorales a los estudiantes.

Los cuatro profesores no tenían cargos titulares y poco después fueron removidos de las cátedras (Partido Socialista, 1945). Pero el insistente malestar universitario convenció al poder ejecutivo de firmar en agosto de 1906 un decreto que modificaba la llamada Ley Avellaneda, que regía los estatutos universitarios. Desde entonces las facultades de la Universidad de Buenos Aires dejaban de estar gobernadas por un consejo formado por miembros vitalicios que no pertenecian al cuerpo de profesores, para comenzar a dirigirse por un consejo de profesores titulares que asumian un cargo periódico y renovable. Ello buscaba limitar la formación de camarillas que pudieran gobernar desde criterios poco académicos, pero se trataba de una modificación que no solo estaba lejos de introducir la democracia universitaria sino que además no simpatizaba con una Argentina democrática. En efecto, el decreto respondía a la preocupación de la fracción "reformista" de la elite gobernante, esto es a las figuras notables que, bajo el protagonismo de Joaquín V. González, asumian que para continuar liderando el proceso modernizador debian iniciar una apertura politica controlada (Zimmerman, 1994). Una clara evidencia de la distancia que mantenía esa fracción de la elite con la democracia es que su reforma más audaz, la reforma electoral a través de la llamada Ley Sáenz Peña, continuaba prescribiendo un ciudadano ideal que legitimaba tanto la jerarquía de la "gente decente" frente a la "plebe" como la "desigualdad social racializada" (Adamovsky, 2009: 58-63). Pero la apertura política traería novedades inesperadas para las distintas fracciones de la elite. Las primeras elecciones presidenciales operaban una transición a una República democrática. A pesar de que el golpe de Estado de 1930 exponía los límites de esa transición y la dejaba trunca, la experiencia abierta en 1916 conseguia reconfigurar las jerarquias sociales (Ansaldi, 2000; Adamovsky, 2009: 93-110, 121- 
134). En cuanto a las universidades, necesitaria del movimiento de la Reforma para desplazar a la elite oligárquica de los gobiernos universitarios. Antes de detenernos en ese proceso, revisemos la sociabilidad estudiantil de los años previos.

\section{Reforma universitaria para consolidar una elite oligárquica americana}

Durante el ciclo de protestas estudiantiles porteñas de 1903-1906, los jóvenes comenzaron a gestionar la personería jurídica de sus centros estudiantiles. El reconocimiento legal de una asociación de carácter gremial en cada facultad otorgaba mayor audibilidad a los reclamos relativos a los aranceles y exámenes presentados a las autoridades. Antes de iniciada la década del 10, todas las facultades de las universidades de Buenos Aires, Córdoba y La Plata contaban con un Centro de Estudiantes reconocido jurídicamente al tiempo que algunos colegios nacionales también tenian ese tipo de centros. ${ }^{6}$

Una vez implementadas las reformas universitarias de 1906, los centros porteños se orientaron más fuertemente a la construcción de instancias recreativas y a medidas dirigidas expresamente a la juventud estudiantil. Además de representar a los estudiantes ante las autoridades universitarias, garantizaron el acceso a los materiales de estudio. Para ello fundaron bibliotecas, promovieron la traducción de textos y editaron folletos y boletines. Cada centro publicó una revista, muchas veces financiada por la facultad, en la que publicitaba sus actividades sociales, como los banquetes en honor a los egresados, y reproducía los textos con los que los estudiantes debian preparar los exámenes (Carreño, 2018).

En varias oportunidades los estudiantes proyectaron la construcción de una instancia articuladora de los distintos centros. En junio de 1908 fundaron la Junta Universitaria Provisoria, convertida tres meses después en la Federación Universitaria de Buenos Aires (FUBA) -vigente en la actualidad-. En esta fundación era decisivo el viaje a Montevideo que había realizado en enero de ese año la treintena de estudiantes argentinos que asistió al Primer Congreso Internacional de Estudiantes Americanos.

El congreso se desarrolló en Montevideo bajo la organización de la Asociación de los Estudiantes de Montevideo. Esta había sido fundada

6. Bajo el impulso de González, en 1905 se había nacionalizado la universidad platense. Esta crecería en los siguientes años desplegando un perfil profesionalista y uno -más debil- científico. Su propósito fue formar tanto a la elite política como a los técnicos ligados al desarrollo económico regional (Biagini, 2001; Graciano, 2008). 
en 1893 por un grupo de estudiantes de la Universidad de la República -y fue renombrada en 1909 como Federación de los Estudiantes del Uruguay-. Hacia 1905 registró un nuevo impulso, cuando encabezó una serie de protestas contra la asistencia obligatoria y los criterios de aprobación de los exámenes y fundó el periódico mensual Evolución (Oddone y Paris de Oddone, 2010). El primer director de Evolución fue Baltasar Brum, un estudiante de derecho que sería presidente del Uruguay en los años en que se expandía la Reforma por el continente, 1919-1923, y que no recogería las demandas democratizadoras del nuevo movimiento estudiantil.

A mediados de 1907, la Asociación difundió tanto entre las agrupaciones estudiantiles de América Latina como entre las de Estados Unidos una convocatoria para participar del Primer Congreso Internacional de Estudiantes Americanos. Los montevideanos consiguieron que en enero del año siguiente llegaran jóvenes que representaban a centros estudiantiles de Argentina, Bolivia, Brasil, Chile, Cuba, Paraguay y Perú. De Estados Unidos solo obtuvieron la adhesión de algunas universidades. La mayoría de los países estuvieron representados por entre 2 y 5 delegados. Uruguay presentó 34 mientras que Brasil llevó 24 y Argentina alcanzó los 38 y fue la encargada de organizar el siguiente Congreso. ${ }^{7}$

Entre los 113 estudiantes americanos que se reunieron en 1908 en Montevideo se encontraba una mujer, la uruguaya Clotilde Luisi. Doctorada en derecho en 1911, la primera abogada del continente se encargó de presentar las bases para la creación de la Liga de Estudiantes Americanos. Esta reunió a las asociaciones estudiantiles del continente entre 1908 y 1914 y logró organizar otros dos encuentros, el de Buenos Aires en 1910 y el de Lima en 1912. El temario de los tres congresos y las actividades que los acompañaron no dejan dudas de que la Liga tramaba una sociabilidad que, si bien se preocupaba por cuestiones educativas y gremiales, se inscribía decididamente en las Repúblicas oligárquicas. Ello fue tan evidente entre los contemporáneos que en 1914 Juan B. Justo se opuso, desde su condición de diputado socialista, a que el Estado argentino gastara el "dinero del pueblo" en la financiación de los estudiantes que asistirian al frustrado Cuarto Congreso, que se había previsto en Santiago de Chile (Garcia, 2000; Biagini, 2012: 69-76).

La aceptación de una mujer en el primer encuentro implicaba una apertura social y política, pero los otros dos encuentros no contaron con mujeres, a pesar de que cada vez eran más las que realizaban estudios universitarios. Por otra parte, ninguno de ellos cuestionó las

7. Esa gran cantidad de delegados seguramente respondió al financiamiento otorgado por el gobierno y a la cercanía geográfica, pues no asistió ningún estudiante de la distante Universidad Provincial de Tucumán ni de la Universidad Nacional de Córdoba. 
restricciones oligárquicas impuestas por las elites que gobernaban el continente. Es más, en el Primer Congreso la delegación estudiantil brasileña buscó -sin éxito- que la Liga diera su pésame al asesinato del Rey de Portugal. Al igual que en los siguientes, en ese primer encuentro las delegaciones estudiantiles fueron recibidas por el gobierno uruguayo como embajadoras intelectuales de sus respectivas repúblicas oligárquicas, e incluso la municipalidad les organizó un garden party con el presidente del Uruguay, Claudio Williman. Para confirmar esa condición de representantes de las repúblicas oligárquicas, los estudiantes aprobaron en la primera sesión del Congreso el nombramiento como "Presidentes Honorarios" del presidente del Uruguay, del Ministro de Relaciones Exteriores y de todos los presidentes de las Repúblicas que habian enviado una delegación o una adhesión. Asimismo, les encargaron al rector de la Universidad de la República, Francisco Soca, y a algunos decanos los discursos de cierre del Congreso.

Pero el posicionamiento politico realizado por esta naciente sociabilidad estudiantil se advertía no solo en las amplias simpatías hacia las repúblicas oligárquicas, sino también en la decisión de englobar a latinos y estadounidenses en el colectivo "americano", pues esta acarreaba la decisión de construir una identidad estudiantil que, a diferencia de la surgida en 1918, no se preocupaba por la denuncia del imperialismo estadounidense. Desde la participación de Estados Unidos en la guerra de la independencia cubana en 1898, nadie podía dudar de su presión política, militar y económica sobre los países latinoamericanos. A las noticias de la prensa continental y las denuncias del poeta y politico cubano José Martí se sumaba en 1900 el Ariel del escritor y político uruguayo José Enrique Rodó. Ese ensayo sistematizó los tópicos de un "antiimperialismo cultural" que tuvo una intensa circulación entre los jóvenes latinoamericanos (Terán, 2008), pero que no fue retomado por la Liga Americana.

Entre 1909 y 1910 varios estudiantes porteños volverian a manifestarse en las calles en defensa de un patriotismo oligárquico y belicista, que reaccionaba contra los inmigrantes anarquistas y socialistas que reclamaban mejoras en las condiciones de trabajo. Este patriotismo se concilió con el americanismo de la Liga. En efecto, el Segundo Congreso se realizó en una Buenos Aires que festejaba su Centenario de la Revolución de Mayo e intentaba estar en la vidriera del mundo como una de las más modernas capitales, pero para ello debía apelar al estado de sitio ya que las huelgas obreras y la "propaganda por los hechos" de los anarquistas desdecían la armonía de esa modernidad. Esa conflictividad social obligó al congreso estudiantil a pronunciarse sobre la existencia de una "cuestión obrera" y los estudiantes decidieron que los reclamos, en general, eran válidos, pero la huelga -a la que otros estudiantes de 
Buenos Aires y Montevideo habían apelado en años anteriores- no era un legítimo método de reclamo.

Otra novedad que se introdujo en el Congreso de 1910 fue la discusión sobre la "extensión universitaria", esto es, sobre instancias en las que las universidades difundieran conocimientos a los obreros que no tenían tiempo ni dinero para una instrucción sistemática. El cordobés Saúl Taborda (entonces un estudiante de abogacía de La Plata y en los años siguientes un destacado líder reformista, ensayista y pedagogo) fue el delegado que en el Segundo Congreso impulsó de modo más entusiasta la extensión. En 1908 el presidente de la Universidad de La Plata, Joaquín V. González, promovió la llegada de Rafael Altamira para impartir lecciones sobre metodologia de la historia y replicar el proyecto de extensión de la Universidad de Oviedo. Entonces se fundó una Universidad Obrera, y esa iniciativa fue continuada al año siguiente por su colega Adolfo Posadas (Vallejo, 2007: 287-292). Taborda buscó que la Liga emprendiera un proyecto similar pero no logró la aprobación de los delegados. Y en 1918 volvía a defender el proyecto, en este caso a través del estudiante de ficción, Víctor Ferro. En su novela Julián Vargas, Ferro inicia una universidad popular y para justificarla propone una argumentación que troca el desprecio al pueblo que tenía la "gente decente" -entre la que se inscribian los estudiantes- por una denuncia, de resonancias anarquistas, contra el Estado y sus hipócritas intelectuales:

Fíjese usted que en las fábricas y en los talleres numerosos obreros desean, ansian aprender alguna cosa, emplear sus ocios en estudiar algo que les ilumine la inteligencia. Han esperado hasta hoy que las universidades del Estado les llamen y les abran sus puertas, pero eso no ocurrirá. Los intelectuales del país entero solo se acuerdan del pueblo en épocas de elecciones. Pasado ese momento le olvidan y le escarnecen. Es una injusticia, señor Vargas; el pueblo tiene alma; crea, trabaja, produce, pero también tiene sueños, quimeras y una sed infinita de ideal. (Taborda, 1918: 240-241)

Para que este tipo de concepciones sean embanderadas por un número importante de estudiantes latinoamericanos habrá que esperar al estallido de la Reforma en Córdoba y a su expansión por las distintas ciudades universitarias del continente. En efecto, mientras que en 1912 varias de las discusiones del Tercer Congreso se preocuparon por la capa y el himno distintivos de los estudiantes, desde el estallido de la Reforma surgía una minoria estudiantil que buscaba que los reclamos gremiales estudiantiles se entrelazaran con los reclamos de los obreros y con ello iniciaban una sociabilidad inscripta decididamente en las izquierdas. 
La Liga debía juntarse en 1914 en Santiago de Chile pero el Cuarto Congreso se suspendió y la Liga se disolvió. Su última actividad fue la presencia, a través de una delegación de estudiantes brasileños y argentinos, en 1913 en el Congreso de la Federación Internacional de Estudiantes "Corda Frates", desarrollado en Ithaca, Nueva York. ${ }^{8}$ Allí la Liga consiguió ser incorporada a la Federación y las tapas de las revistas de los distintos Centros de Estudiantes expusieron desde entonces y por algunos años la guarda que identificaba la adhesión a la Federación.

Los estudiantes latinoamericanos recién volverian a reunirse en un congreso internacional en 1921, cuando la Revolución Mexicana los convocaba a discutir cuestiones gremiales y politicas en un continente que ya contaba con varias repúblicas democráticas, con movimientos obreros que participaban de la "hora emancipatoria excepcional" que habría abierto la Revolución Rusa y con diversos grupos estudiantiles disputando una identidad nacionalista o una izquierdista de la Reforma Universitaria.

\section{Reforma universitaria para participar del movimiento mundial emancipatorio}

El 15 de junio de 1918 un grupo de estudiantes y graduados irrumpía en la elección del rector de la Universidad Nacional de Córdoba e iniciaba una toma que sería identificada como el inicio simbólico de la Reforma universitaria. Los jóvenes reaccionaban tanto contra un gobierno universitario de carácter vitalicio y corporativo como contra una enseñanza impartida por profesores poco calificados y adscriptos al clericalismo conservador. Si bien esos estudiantes recogian las demandas gremiales que venían formulándose desde hacía décadas, participaban de un álgido ciclo de agitación liberal local (Agüero, 2016). Y poco después iniciaban una solidaridad obrera que marcaría una significativa ruptura con la sociabilidad estudiantil previa.

Seis días después de la toma, la Federación Universitaria de Córdoba (FUC) lanzaba un nuevo número de La Gaceta Universitaria. Este ponía a circular el célebre "Manifiesto liminar", reclamaba una nueva intervención y convocaba a un acto, a realizarse el 23 de junio, que

8. Esta federación fue fundada en 1898 en Turín por una delegación de estudiantes de diversas universidades europeas. Su objetivo fue la promoción de la fraternidad estudiantil, pero no logró una organización periódica y, tras una primera vinculación con el fascismo italiano, se disolvió a fines de la década del 20. Su nombre coincide casualmente con la agrupación de profesores católicos y antirreformistas cordobeses actuante en 1918. Sobre la participación de la Liga en el encuentro de Ithaca, contamos con la crónica de Arturo Capdevila, quien asistió como estudiante y publicó el relato cuando ya se había convertido en un reconocido escritor (Capdevila, 1933). 
contaria entre sus oradores con Alfredo Palacios, el socialista que portaba la aureola de haber sido el primer diputado de América Latina. E1 diario clerical conservador de Córdoba, Los Principios, caracterizaba al masivo acto como "un mitin socialista anticlerical" y seguramente no exageraba, pues la crónica informaba que los jóvenes federados cantaron La Marsellesa, el himno de la Revolución Francesa, y el Himno de los trabajadores, que solian entonar los obreros socialistas. ${ }^{9}$

El Manifiesto había sido redactado por uno de los líderes de la Asociación Córdoba Libre, el joven abogado Deodoro Roca, pero llevaba la firma de la Junta Directiva de la FUC. ${ }^{10}$ En una prosa sumamente estetizada que recordaba al ensayo Ariel de Rodó, los jóvenes se dirigían "a los hombres libres de Sudamérica" para narrarles los acontecimientos que llevaron a la toma de la Universidad y convocarlos a "recoger la lección" y "colaborar en la obra de libertad que se inicia". La toma habría roto "la última cadena que en pleno siglo XX nos ataba a la antigua dominación monárquica y monástica" y sería la primera batalla ganada en un combate a favor de una universidad laica y democrática, combate que pronto se ligaria a la exigencia de una sociedad más igualitaria.

La FUC reprodujo en centenas el "Manifiesto liminar" y a través de sus representantes consiguió que a los pocos días llegara a los universitarios y estudiantes secundarios de las distintas ciudades argentinas así como a los jóvenes de Montevideo, Santiago de Chile y Lima. Los cordobeses sabian que el éxito de su programa renovador dependía de la red de apoyo que lograran tramar y los periódicos estudiantiles junto a las llamadas, telegramas y cartas se convirtieron en vías decisivas para construir una sociabilidad estudiantil masiva que adquiría escala latinoamericana y por primera vez se enfrentaba a las elites oligárquicas (Bergel y Martínez Mazzola, 2010).

Las diferencias entre la impronta clerical-conservadora que tenían muchos cursos cordobeses y la enseñanza moderna y laica que recibían los estudiantes porteños y platenses no impidieron que la estudiantina trazara lazos de solidaridad a través de la fundación de la primera agrupación nacional, la Federación Universitaria Argentina (FUA). Esta se fundó en abril, el mismo día en que el presidente nacional Yrigoyen decretaba la intervención de la Universidad de Córdoba. La toma del rectorado en junio marcaba el fracaso de la intervención. Al mes siguiente se reunian por primera vez los delegados de las cinco universidades nacionales para discutir problemas gremiales y sociales en el Primer Congreso

9. "La manifestación de la Federación Universitaria resulta un mitin socialista anticlerical", Los Principios, 26 de junio de 1918.

10. Sobre la importancia de esta Asociación en las multitudinarias manifestaciones reformistas que se realizaron en Córdoba durante 1918, véase Agüero (2016). 
Nacional de Estudiantes. La minoría simpatizante de las izquierdas no lograba que las federaciones gremiales realizaran un pronunciamiento explícito vinculado a la profundización de una República democrática. El Congreso formó una comisión que en los meses siguientes reclamó con éxito la nacionalización de la Universidad Provincial de Santa Fe, que se convertiria en la Universidad del Litoral, y de la Universidad de Tucumán. Pero las resoluciones fueron decepcionantes para los delegados que buscaban ligar la identidad estudiantil tanto a la República democrática como a un régimen político orientado por la justicia social y la igualdad económica. En efecto, el proyecto de enseñanza gratuita presentado por el estudiante de ingenieria Gabriel de Mazo -quien presidiría la FUA en 1920- así como los proyectos que -orientados por la prédica de Roca, Taborda e Ingenieros- vinculaban los cambios educativos y los lazos latinoamericanos al horizonte emancipatorio abierto por la Revolución Rusa no lograron la aprobación. ${ }^{11}$ Ello no disuadía a esa minoría de construir una nueva identidad en la que la reforma gremial se unía a la reforma social e inauguraba la "Reforma Universitaria", sino que la obligaba a recorrer otros caminos, al punto que el Segundo Congreso Nacional de Estudiantes recién se realizaria en 1932.

Cuando estalla la Reforma, hacía un año que la "revolución social" preocupaba al mundo y que el vocabulario politico había incorporado los términos "bolchevique", "maximalismo" y "soviet". Pocos lograron permanecer indiferentes a esa revolución que, bajo el liderazgo de Lenin y Trotsky, prometía las reivindicaciones obreras "máximas" y anunciaba la expansión mundial de una era de igualdad social y emancipación proletaria. El éxito revolucionario y la sucesión de insurrecciones europeas renovaban, a escala mundial, las polémicas e iniciativas tanto de las derechas como de las izquierdas. Entre las primeras cundió el temor ante lo que entendian como el contagio social de un peligroso desorden místico. Entre las segundas, en cambio, se registró un fuerte debate sobre la vía revolucionaria y el centralismo estatal, que terminaria saldándose con la incorporación de dos nuevos miembros a la conflictiva familia de las izquierdas: a distancia del anarquismo y del socialismo se sumaban el anarcobolchevismo y el comunismo bolchevique.

En una escala menor que en Alemania, España, Hungria e Italia, se abría en Argentina un trienio rojo marcado por el creciente conflicto social -cuya expresión más violenta sería la represión a los obreros huelguistas y a los judíos protagonizada por policias y civiles en enero de 1919- y el entusiasmo revolucionario de socialistas y anarquistas

11.Todos los proyectos discutidos pueden consultarse en Del Mazo (1927, t. II). Sobre el apoliticismo y el moderatismo predominantes en los estudiantes de la Universidad de Buenos Aires, véase Buchbinder (2008). 
que se reconocian bolcheviques (Doeswijk, 2013; Camarero, 2017). Y una minoria de reformistas buscaría que también la "juventud culta" participara de la ola maximalista. Para ello a mediados de 1919 el grupo estudiantil más numeroso de Buenos Aires, el Ateneo de Estudiantes Universitarios (1914-1920), abandonó su "pacto pluralista" y se declaró "del lado de los oprimidos" y su lucha emancipatoria. Entonces dejaba de editar la revista cultural Ideas (1914-1919) para poner a circular el periódico filobolchevique Clarín (1919-1920). La misma definición estudiantil era propuesta por la revista socialista Bases, que fundó en mayo de 1919 el joven Juan Antonio Solari. Y pronto esta "tribuna de la juventud" fue identificada como la hermana mayor de una serie de revistas filobolcheviques que se propusieron inscribir a la Reforma en el movimiento emancipatorio mundial: en Córdoba, Roca, Taborda, Emilio Biagosch y otros líderes reformistas editaron Mente (1920), en Rosario los jóvenes del Centro Evolución crearon Verbo Libre (1920) y luego estudiantes secundarios vinculados al Centro fundaron La Antorcha (1921-1922) y Germinal (1922-1923), La Plata también tuvo su Germinal estudiantil (1919-1921) y en Buenos Aires le sucedió a Bases la mítica Insurrexit (1920-1921) (Bustelo y Dominguez Rubio, 2017; Tarcus, 2004).

Los estudiantes que editaban esas revistas invitaron a los obreros sindicalizados a sus charlas y conferencias -en convocatorias que por primera vez tendian a desjerarquizar el vínculo- al tiempo que se solidarizaron con las protestas y huelgas obreras y buscaron la adhesión de los sindicatos obreros a sus reclamos. En 1920 intentaron consolidar la nueva identidad estudiantil a través de una breve Federación de Estudiantes Revolucionarios, fundada en Rosario. Otro intento de inscribir la Reforma en la Revolución fue encabezado por la FUC, que desde mediados de 1918 habia expresado su solidaridad obrera a partir de su vinculación a la Federación Obrera Local, de tendencia socialista bolchevique, y por la dirección de la FUA, que entre 1919 y 1922 intentó que esa solidaridad se extendiera al movimiento estudiantil nacional. Ninguna de esas iniciativas radicalizadas logró consolidar una plataforma política prolongada, pero ello no impidió que en 1918 la gran prensa difundiera pruebas -que poco después se revelaron falsas- sobre un "complot maximalista" tramado entre la FUC y la federación obrera ni que dos años después un comisario platense realizara la misma acusación, en este caso a los dirigentes de la FUA y de la FORA anarquista. ${ }^{12}$

La FUA sesionaba en Buenos Aires, se componía de dos delegados por cada una de las federaciones regionales y preveía que las univer-

12. "Encuesta de Vida Nuestra sobre la situación de los judíos en la Argentina. Respuesta de Enrique Barros", Vida Nuestra, n 9, marzo de 1919, pp. 197-198; "Aquel complot maximalista", La Voz del Interior, 24 de abril de 1920, p. 3. 
sidades distantes de Buenos Aires estuvieran representadas a través de estudiantes porteños. Esta cláusula les permitió a los izquierdistas porteños erigirse en delegados regionales e imprimirle a la Federación y a su publicación, el Boletín de la FUA (1920-1923), un sesgo obrerista que confluyó con la FUC pero que entró en fuerte rivalidad con la FUBA. En efecto, esta rechazó la solidaridad obrera y llamó a circunscribir la acción estudiantil a las cuestiones gremiales, e incluso en 1919 hizo público su malestar con la FUA porque esta no sancionaba las "actividades no universitarias" de la federación cordobesa y al año siguiente decidió su separación de la FUA (Del Mazo, 1976: 101).

Hacia 1923 el horizonte revolucionario se alejaba. Las insurrecciones europeas habian sido derrotadas y la economía argentina se recuperaba junto al descenso del conflicto social. El movimiento reformista argentino proponia otras identidades políticas pero continuaba enfrentado a un orden oligárquico. Los líderes estudiantiles Horacio Valdés en Córdoba y Julio V. González en Buenos Aires encabezaban un breve intento de inscribir la Reforma en una clase media que, guiada por el Partido Demócrata Progresista, debía evitar la confrontación entre trabajadores y empresarios, mientras que Alejandro Korn orientaba al grupo platense que se vinculaba con un "socialismo ético" y, bajo el magisterio de Ingenieros y Palacios y el despliegue de la Unión Latino Americana, se delineaba una identidad latinoamericana y antiimperialista que estrechaba vínculos continentales y se volvería característica de la Reforma Universitaria.

La prolongación del conflicto estudiantil cordobés en un movimiento político-cultural latinoamericano ya había construido una sociabilidad estudiantil marcada por una nueva figura de estudiante. Frente al "niño bien" al que la universidad le permitía confirmar -o alcanzar- su pertenencia a la "gente decente", comenzaba a aparecer un estudiante que, a distancia de los partidos políticos, se inscribía en una cultura de izquierdas. Asimismo, en rivalidad con el intento de ceñir la Reforma a las cuestiones gremiales o de inscribirla en un orden oligárquico, aquel estudiante se comprometía con la emancipación del género humano, sea a través de una ciencia que remediara las injusticias sociales, de proyectos de extensión universitaria o de lazos de solidaridad obrero-estudiantil. Y, como precisa Portantiero, la Reforma pronto se expandía en las diversas ciudades de América Latina y en varias de ellas no solo conformaba una "escuela ideológica para los sectores avanzados de la pequeña burguesía” (Portantiero, 1978: 14), sino también lograba fundar estructuras políticas que incidian de modo directo en el orden social.

Para concluir subrayemos que, a distancia de la sociabilidad estudiantil construida entre 1872 y 1914, la que se inició en 1918 cuestionaba la condición de las universidades como espacios de formación de 
las elites oligárquicas y, si retoma los reclamos de democratización de las universidades de las décadas anteriores, lo hace para vincularlos a la democratización de toda la sociedad y a un latinoamericanismo que denunciaba el imperialismo estadounidense.

En países latinoamericanos -como Perú y Cuba- en los que aún no se había realizado una transición a una república democrática ni existía un sistema de partidos politicos desarrollado, la Reforma convertía a los estudiantes en organizadores de movimientos politicos masivos: el estudiante Víctor Raúl Haya de la Torre junto a otros estudiantes fundarían el partido que a lo largo del siglo XX aglutinó a los sectores medios peruanos, el APRA, mientras que el estudiante Julio Antonio Mella lideraría la creación del Partido Comunista Cubano, que sería una referencia central de las luchas politicas de las décadas siguientes. En Argentina, en cambio, la contraelite reformista se incorporaba a distintos partidos ya existentes, sobre todo a partir de la interrupción de la República democrática que produjo el golpe de Estado de 1930, y era una de las constructoras del movimiento antifascista, desde el que a partir de 1945 se enfrentaría al peronismo. Más allá de ello la incorporación de los reformistas a los partidos políticos iniciaba la disputa -que llega hasta nuestros dias- sobre la auténtica filiación del movimiento reformista en la Unión Cívica Radical, el Partido Socialista o el Comunista.

\section{Bibliografia}

Adamovsky, Ezequiel (2009), Historia de la clase media argentina. Apogeo y decadencia de una ilusión, 1919-2003, Buenos Aires: Planeta.

Agüero, Ana Clarisa (2016), "Córdoba. 1918, más acá de la reforma", en Adrián Gorelik y Fernanda Arêas Peixoto (eds.), Ciudades sudamericanas como arenas culturales, Buenos Aires: Fondo de Cultura Económica, pp. 96-115.

Ansaldi, Waldo (2000), "La trunca transición del régimen oligárquico al régimen democrático", en Ricardo Falcón (ed.), Nueva historia argentina, t. VI: Democracia, conflicto social y renovación de ideas, Buenos Aires: Sudamericana, pp. 15-57.

Barrancos, Dora (2007), Mujeres en la sociedad argentina. Una historia de cinco siglos, Buenos Aires: Sudamericana.

Bergel, Martín y Ricardo Martínez Mazzola (2010), "América Latina como práctica. Modos de sociabilidad intelectual de los reformistas", en Carlos Altamirano (ed.), Historia de los intelectuales en América Latina, t. II, Buenos Aires: Katz, pp. 119-145.

Biagini, Hugo (2012), La contracultura juvenil. De la emancipación a los indignados, Buenos Aires: Capital Cultural.

- (comp.) (2001), La Universidad de La Plata y el movimiento estudiantil. Desde sus orígenes hasta 1930, La Plata: Edulp. 
Bianchi, Alfredo (1932), Veinticinco años de vida intelectual argentina, Buenos Aires: Nosotros.

Bioy, Adolfo (1963), Años de mocedad, Buenos Aires: Nuevo Cabildo.

Botana, Natalio y Ezequiel Gallo (1997), De la República posible a la República verdadera, Buenos Aires: Ariel.

Buchbinder, Pablo (2005), Historia de las universidades argentinas, Buenos Aires: Sudamericana.

- (2008), ¿Revolución en los claustros? La Reforma universitaria de 1918, Buenos Aires: Sudamericana.

Bustelo, Natalia y Lucas Dominguez Rubio (2017), "Radicalizar la Reforma universitaria. La fracción revolucionaria del movimiento estudiantil argentino (1918-1922)", Anuario Colombiano de Historia Social y de la Cultura, vol. 44, $\mathrm{n}^{\circ}$ 2, Bogotá. Disponible en http://www.revistas.unal. edu.co/index.php/achsc.

Camarero, Hernán (2017), Tiempos rojos. El impacto de la Revolución rusa en la Argentina, Buenos Aires: Sudamericana.

Capdevila, Arturo (1933), Una estudiantina de hacha y tiza, Buenos Aires: en Selección. Cuadernos mensuales de cultura.

Carreño, Luciana (2018), "Los caminos de la Reforma Universitaria. Sociabilidad y vida estudiantil en los centros de estudiantes de la Universidad de Buenos Aires (1900-1918)", en Quinto Sol, vol. 22, n 1.

Del Mazo, Gabriel (1976), Vida de un politico argentino. Convocatoria de recuerdos, Buenos Aires: Plus Ultra.

- (ed.) (1927), La Reforma universitaria, 6 vols., Buenos Aires: Círculo Médico Argentino y Centro de Estudiantes de Medicina, FUBA.

Devoto, Fernando (2002), Nacionalismo, fascismo y tradicionalismo en la Argentina moderna. Una historia, Buenos Aires: Siglo XXI.

Doeswijk, Andreas (2013), Los anarco-bolcheviques rioplatenses, Buenos Aires: Cedinci.

Gálvez, Manuel (2003), Recuerdos de la vida literaria, vol. 1: Amigos y maestros de mi juventud en el mundo de los seres ficticios, Buenos Aires: Taurus.

García, Susana V. (2000). "Embajadores intelectuales. El apoyo del Estado a los congresos de estudiantes americanos a principios del siglo XX", Estudios Sociales, $\mathrm{n}^{\circ} 19$, Santa Fe, pp. 65-84.

Graciano, Osvaldo (2008), Entre la torre de marfil y el compromiso politico. Intelectuales de la izquierda argentina, 1918-1955, Bernal: Editorial UNQ.

Halperín Donghi, Tulio (1962), La Universidad de Buenos Aires, Buenos Aires: Eudeba.

Lazarte, Juan (1935), Lineas y trayectorias de la Reforma Universitaria, Córdoba: Librería Ruiz.

Oddone, Juan y M. Blanca Paris de Oddone (2010), Historia de la Universidad de la República, tomo II: La Universidad, del militarismo a la crisis, Montevideo: Ediciones Universitarias.

Ortiz, Tulio y Luciana Scotti (s/d), "Las Reformas antes de la Reforma. Pri- 
meros movimientos estudiantiles en la Universidad de Buenos Aires", en http://www.uba.ar/reforma/download/reformas.pdf.

Partido Socialista (1945), La Reforma Universitaria y el Partido Socialista, Buenos Aires: Partido Socialista.

Portantiero, Juan Carlos (1978), Estudiantes y politica en América Latina (1918-1938), El proceso de la Reforma Universitaria, México: Siglo XXI.

Rock, David (2006), La construcción del Estado y los movimientos políticos en la Argentina, 1860-1916, Buenos Aires: Prometeo.

Rojkind, Inés (2012), "El gobierno de la calle. Diarios, movilizaciones y politica en el Buenos Aires del novecientos", en Secuencia. Revista de historia y ciencias sociales, $\mathrm{n}^{\circ}$ 84, México, pp. 97-123. Disponible en http://www.redalyc.org/pdf/3191/319128360005.pdf.

Suriano, Juan (2004), Anarquistas. Cultura y politica libertaria en Buenos Aires, 1890-1910, Buenos Aires: Manantial.

Taborda, Saúl (1918), Julián Vargas, Córdoba: Elzeviriana.

Tarcus, Horacio (2004), "Revistas, intelectuales y formaciones culturales izquierdistas en la Argentina de los veinte", Revista Iberoamericana, $\mathrm{n}^{\circ}$ 208-209, Madrid, pp. 749-772.

- (dir.) (2007a), Diccionario biográfico de la izquierda argentina. De los anarquistas a la "nueva izquierda" (1870-1976), Buenos Aires: Emecé.

- (2007b), Marx en la Argentina. Sus primeros lectores obreros, intelectuales $y$ científicos, Buenos Aires: Siglo XXI.

Terán, Oscar (2008), Historia de las ideas en la Argentina. Diez lecciones iniciales, 1810-1980, Buenos Aires: Siglo XXI.

Vallejo, Gustavo (2007), Escenarios de la cultura cientifica argentina. Ciudad y universidad (1882-1955), Madrid: Consejo Superior de Investigaciones Científicas.

Viñas, David (1964), Literatura argentina y realidad politica, Buenos Aires: Jorge Álvarez.

Zimmerman, Eduardo (1994), Los liberales reformistas. La cuestión social en la Argentina 1890-1916, Buenos Aires: Sudamericana.

$$
* * *
$$

Titulo: From the repudiation of bad teachers to social emancipation. The claims of Buenosairean students (1872-1930)

Resumen: El artículo recorre los primeros reclamos de los estudiantes de la ciudad de Buenos Aires por una "reforma universitaria", desde la fundación en 1872 de la Asociación 13 de diciembre hasta la articulación en 1918 del movimiento político-cultural de la Reforma Universitaria, pasando por el ciclo de huelgas estudiantiles de 1903-1906 y el desarrollo de la Liga de Estudiantes Americanos entre 1908 y 1914. El recorrido se propone mostrar que si bien puede reconocerse cierta continuidad en los reclamos gremiales asociados a una "reforma universitaria", el movimiento estudiantil que comenzó a estructurarse 
en 1918 realizó una decisiva ruptura política: dejó de legitimar un orden social oligárquico para acercarse a las izquierdas y su lucha emancipatoria.

Palabras clave: Reforma Universitaria - estudiantes argentinos - República oligárquica - izquierdas

\begin{abstract}
The paper looks over the first student protests in Buenos Aires for a "university reform", since the foundation of the Asociacion 13 de diciembre in 1873 until the articulation of the political-cultural movement of the Reforma Universitaria in 1918, through the cycle of student strikes in 1903-1906 and the development of the Liga de Estudiantes Americanos between 1908 and 1914. The purpose is to show that although it can recognize any continuity in the gremial demands associated to a "university reform", the student movement that begins in 1918 makes a crucial political breakup: it separates to an oligarchic Republic to closer to the lefts and its struggle for the emancipation.

Keyboards: University Reform - Argentinian students - oligarchic republic - lefts
\end{abstract}

Recepción: 6 de febrero de 2018. Aprobación: 5 de marzo de 2018. 